\title{
Will Systems Biology Deliver Its Promise and Contribute to the Development of New or Improved Vaccines?
}

\section{What Really Constitutes the Study of "Systems Biology" and How Might Such an Approach Facilitate Vaccine Design}

\author{
Ronald N. Germain \\ National Institute of Allergy and Infectious Diseases, National Institutes of Health, Bethesda, Maryland 20892 \\ Correspondence: rgermain@nih.gov
}

A dichotomy exists in the field of vaccinology about the promise versus the hype associated with application of "systems biology" approaches to rational vaccine design. Some feel it is the only way to efficiently uncover currently unknown parameters controlling desired immune responses or discover what elements actually mediate these responses. Others feel that traditional experimental, often reductionist, methods for incrementally unraveling complex biology provide a more solid way forward, and that "systems" approaches are costly ways to collect data without gaining true insight. Here I argue that both views are inaccurate. This is largely because of confusion about what can be gained from classical experimentation versus statistical analysis of large data sets (bioinformatics) versus methods that quantitatively explain emergent properties of complex assemblies of biological components, with the latter reflecting what was previously called "physiology." Reductionist studies will remain essential for generating detailed insight into the functional attributes of specific elements of biological systems, but such analyses lack the power to provide a quantitative and predictive understanding of global system behavior. But by employing (1) large-scale screening methods for discovery of unknown components and connections in the immune system (omics), (2) statistical analysis of large data sets (bioinformatics), and (3) the capacity of quantitative computational methods to translate these individual components and connections into models of emergent behavior (systems biology), we will be able to better understand how the overall immune system functions and to determine with greater precision how to manipulate it to produce desired protective responses.

\section{GREAT DEBATES}

What are the most interesting topics likely to come up over dinner or drinks with your colleagues? Or, more importantly, what are the topics that don't come up because they are a little too controversial? In Immune Memory and Vaccines: Great Debates, Editors Rafi Ahmed and Shane Crotty have put together a collection of articles on such questions, written by thought leaders in these fields, with the freedom to talk about the issues as they see fit. This short, innovative format aims to bring a fresh perspective by encouraging authors to be opinionated, focus on what is most interesting and current, and avoid restating introductory material covered in many other reviews.

The Editors posed 13 interesting questions critical for our understanding of vaccines and immune memory to a broad group of experts in the field. In each case, several different perspectives are provided. Note that while each author knew that there were additional scientists addressing the same question, they did not know who these authors were, which ensured the independence of the opinions and perspectives expressed in each article. Our hope is that readers enjoy these articles and that they trigger many more conversations on these important topics.

Editors: Shane Crotty and Rafi Ahmed

Additional Perspectives on Immune Memory and Vaccines: Great Debates available at www.cshperspectives.org

Copyright $\odot 2018$ Cold Spring Harbor Laboratory Press; all rights reserved; doi: 10.1101/cshperspect.a033308

Cite this article as Cold Spring Harb Perspect Biol 2018;10:a033308 
$\mathrm{O}$ ver the past decade, substantial attention has been given to the use of "systems biology" to enhance our understanding of immunity and potentially improve the design of new vaccines that will overcome existing limitations with regard to potency, durability, and specificity (Germain 2010; Pulendran et al. 2010; Trautmann and Sekaly 2011). Using methods for global transcriptional profiling in particular, the notion of "systems vaccinology" emerged to great fanfare (Querec et al. 2006; Gaucher et al. 2008; Nakaya et al. 2011). But as with any substantial change in the status quo, some are strong advocates of the new approach and its promises (Perrin et al. 2010; Oberg et al. 2011; Li et al. 2013; Hagan et al. 2015), while others suggest that there is less to be gained from such expensive studies than its supporters suggest and argue that traditional investigation is more likely to generate new understanding in a reliable costeffective manner.

What is the "systems biology of vaccines" or "systems vaccinology" and what are the arguments made in favor of its value by its proponents? Other works in the literature will elaborate on this topic in some detail, but the answers can be summarized succinctly. The approach as commonly employed involves a two-step process. First is the application of new methods for large-scale ("omic") data collection involving RNA, proteins, cells, and metabolic signatures to gather an "unbiased" set of information about the baseline and postperturbation state of the immune system. Second is mathematical/ statistical analysis of such data sets to identify correlations between these measured elements and the immune state of an individual, as well as to draw inferences about connectivity within the complex set of components that were measured. The benefits are proposed to be a lessbiased and more complete analysis of what regulates immune responses and what aspects of host defense contribute to vaccine efficacy or resistance to damaging infection (Perrin et al. 2010; Oberg et al. 2011; Li et al. 2013; Hagan et al. 2015). The approach is presumed to permit discovery of molecules or cells that play previously unrecognized roles in these processes, doing so in an efficient manner that avoids the very long process of narrowly focused iterative experimentation that is the more typical mode of discovery.

The naysayers note that, in the early work in this field, much that was reported involved the application of an expensive genomic method to the rediscovery of what was already known, mainly that plasma cells make antibody and more plasma cells mean more antibody. Further, while some "hairball" or "ridiculome" plots of network connectivity were included in such reports, there was little done to construct inclusive models of immunity based on such connectivity or omic-scale data; the important insights into immunity that emerged from these studies came from what seemed to be cherry-picking among differentially expressed genes and not unbiased, quantitative, and systematic analysis of the data (Fig. 1). These opposing views have left the field in a difficult position, with the "believers" expanding their studies and using more resources in the face of skepticism from many other investigators who see such research as consuming funds better used for more conventional exploration of immunity.

\section{AS USUAL, MISUNDERSTANDINGS GIVE RISE TO THE OPPOSING VIEWS}

I think that much of the problem here comes from a fundamental misunderstanding in the (immunological) research community about what truly constitutes "systems biology" and how this approach can complement the more reductionist type of investigation that has proved so powerful for many decades.

The mere collection of extremely large omic data sets does not constitute a true systems analysis. While important insights can be gained from mining such data sets, the real value of this approach lies in the application of quantitative, integrative methods that seek to discern how many parts work together to give outcomes not obvious from the behavior or properties of each of the elements (Butcher et al. 2004; Germain et al. 2011). The statistical analyses that have been put forward to date as the core of systems vaccinology, while clearly important 
Inputs and outputs of systems biology research

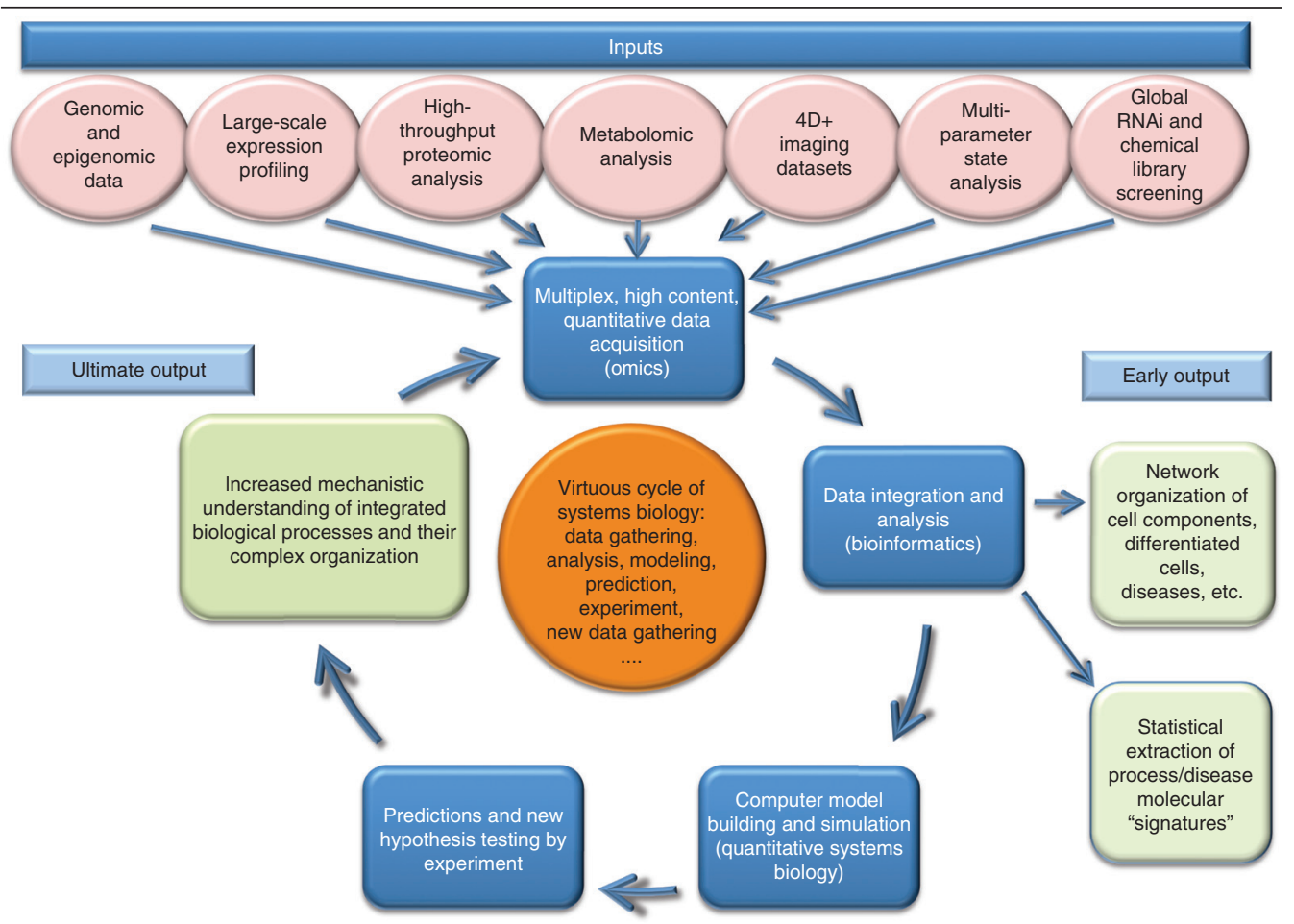

Figure 1. The three major components described in the text as essential to a comprehensive systems biology approach to vaccines are shown centered around the "virtuous cycle" of systems biology research. At the top are a series of omic-scale technologies that collect data on the components of a system. At the right are the early outputs achieved through use of statistical bioinformatics methods, including network relationships and possible biomarkers for diagnosis and prognosis. At the bottom and left side are the parts of computational systems biology that involve mathematical modeling and simulation, prediction, testing of predictions, and when predictions fail (as they always will when tested rigorously), improvement of the model using data from the omic technologies. Testing predictions involves the expertise typically associated with standard laboratory investigation and experiment.

and useful, are only a small part of a comprehensive "systems biology" investigation of immunity. The major products of informatics-based analyses are biomarker signatures of immune state or correlations with outcomes that can be useful clinically for stratification, diagnosis, and prognosis, but they do not provide direct insight into how the host response operates and what crucial molecules or cells need to be targeted in what manner to evoke a desired response. Computational systems biology is a distinct subdiscipline focused on creating (quantitative) models of complex behaviors involving many components, an approach that permits detailed tests of how changes to parameters affect the system's behavior (its "emergent properties"). The latter are precisely what we need if we are to reach the goal of more rational vaccine design, in particular, creation of vaccines that are based on identification of the crucial "choke points" that regulate such processes as $\mathrm{T}$ follicular helper $\left(\mathrm{T}_{\mathrm{FH}}\right)$ generation, germinal center (GC) development, affinity maturation, the choice between memory $B$ cells and plasma cell fate or the long-term survival of plasma cells when humoral immunity is the desired goal and that point us to the control elements for stem memory-cell development, tissue-resident memory cell (TRM) pro- 
duction, effector subtype choice, and related parameters for cell-mediated host defense.

As an example of a question that is best analyzed using the latter type of systems approach, consider the issue of why attenuated viral vaccines are often so much more effective than most combinations of antigen and adjuvant and why virus-like particles seem the exception (Minor 2015; Schiller and Lowy 2015). Is this an effect at the initial antigen-presenting cell level in terms of peptide-major histocompatibility complex ( $\mathrm{pMHC}$ ) molecule ligand display, costimulatory molecule expression, or tissue positioning; is it an effect of Toll-like receptor (TLR) signaling directly involving $\mathrm{T}$ cells and/or B cells and if so through the same or different TLRs for both cell types; is it the result of the immune system having evolved to respond optimally to a type of large-scale pattern recognition that equates infection with a rising antigen load over several days, something achieved with the attenuated vaccines and not with subunit material; is it the combination of cytokines elicited that is distinct from that achieved with adjuvants so far; do these vaccines affect $\mathrm{T}_{\mathrm{FH}}$ quantity, quality, or both, and how do such effects relate to the other parameters I mention; what about the deposition of immune complexes on follicular dendritic cells (FDCs) and the effect of such depots on affinity maturation? What about the density and spacing of determinants seen by $\mathrm{B}$ cells that older studies argued play critical roles in driving B-cell activation and may be characteristic of organized viral capsid proteins (Dintzis et al. 1976)? This listing of just a few of the more obvious sites at which a vaccine must operate to promote an optimal response should make clear that we would benefit tremendously from a more quantitative understanding of how all of these elements fit together in driving a potent long-lived response.

What needs to be recognized is that its name "immune system" is the very essence of why truly systems-level analyses and quantitative models are needed going forward if we are to have a high likelihood of success in designing vaccines that elicit exactly the quality and quantity of response needed for effective immunity. We now know of hundreds, even thousands, of cell types, soluble and cell-bound mediators, and local or long-distance interactions that contribute to host defense, but most studies look at only one or a very few of these parameters at one time. Further, most studies are qualitative rather than quantitative; they test whether a cell or molecule is needed through addition, knockout, or antibody-blocking methods. This is useful information, but it is quite clear that graded changes in the number of a particular cell type or the intracellular concentration of a protein can have profound effects on the behavior of the system (Feinerman et al. 2008; Altschuler and $\mathrm{Wu} 2010$ ). Our thinking tends to be analog or linear, but the extensive number of feedback loops that characterize every level of biology, from molecules, to cells, to tissues and organs, leads to very nonlinear system-level behavior that is almost impossible for us to intuit (Germain 2001). Beyond this, the exponential amplification of small effects through the rapid proliferation of many immune cell types once activated adds further to this gap between qualitative thinking and in vivo biology.

Noting this complexity and nonlinearity of ten engenders a response along the lines of, "Yes, the system has so many components, many still unknown, and such convoluted interactions we could not possibly build a useful model that in any way comes close to what we biologists think is reality, so we should avoid this path and persevere along existing lines, manipulating one or another element, seeing what happens, and gradually gaining more insight." But this view ignores the fact that biologists are always building "models" when they do their research. Each experiment is premised on a hypothesis that involves such a model, perhaps of what cytokine controls what flavor of immune response, or what cells contribute to protection against what organism. The distinction between these models and those employed in a real systems biology approach is that they are typically extremely coarse-grained (lacking many elements) and nonquantitative. The extreme lack of "completeness" and this avoidance of quantitative considerations can severely limit the utility of the results in predicting how the system will behave when faced with a new perturbation (vaccine). For example, some networks in biol- 
Will Systems Biology Develop New or Improved Vaccines?

ogy operate in a nearly digital manner, with limited to no response across a wide range of input and then a maximal response once a threshold is crossed by a small further increase in input. This is quite distinct from more analog responses, where it takes a hundredfold input change to go from modest but detectable output to full response (Altan-Bonnet and Germain 2005; Das et al. 2009; Gottschalk et al. 2016). If we do not know which pattern characterizes any of the processes relevant to a vaccine response, it is nearly impossible to design a suitable vaccine that does not result in a very dichotomous response across a reasonable range of component concentration.

A related point can be made with respect to propagation of small differences across a larger network. If we take the existing paradigm for $\mathrm{T}$ cell-dependent humoral immunity as summarized above, it is clear that small changes in how antigen is delivered to either DCs or B cells, in how activated these presenting cells become and how much pMHC they display, with what timing the activated T cells and B cells move to a small region of the lymph node (LN) or spleen white pulp to become densely enough concentrated to permit useful cell-cell interactions, in how the immunogen localizes to FDCs to support B-cell affinity maturation, or in the balance between memory B-cell formation or plasmacell generation can all have profound effects on whether a vaccine will engender a sufficiently robust response to exceed the threshold for effective resistance to infection and for how long such resistance would last. Models that examine the quantitative relationships that exist between each of these features and the antibody response can reveal which of the steps are potentially rate limiting and what manipulations would best ensure that the outputs of those steps are maximized. Early efforts in this direction have been made with detailed and increasingly useful models of the GC reaction (Kleinstein and Jaswinder 2001; Meyer-Hermann 2006; Tas et al. 2016), but we are far from creating such quantitative dynamic models for all the other aspects of humoral responses that I have mentioned here, no less an integrated model that links the different processes together.

\section{POTENTIAL ROADBLOCKS AND HOW TO OVERCOME THEM}

Skeptics about the value of systems approaches make valid points when they note the large number of gaps in our knowledge, the substantial lack of the necessary quantitative data sets, and the daunting nature of building models that are true to the known biology rather than highly abstracted constructs. But there are two divergent potential responses to these concerns. The first is to decide that the barriers to useful modeling and quantitative systems analyses are so high that it would be impractical to work to reduce them and that we should proceed as we have for many decades, perhaps guided by a bit more insight from omic data collection and statistical processing. I would argue that is the wrong response and suggest instead that we should examine what information, measurements, and modeling tools are lacking and why, then seek to empower investigators (and more likely, groups of investigators) to attack these limitations and move to a future in which the barriers have been eliminated and true systems biology methods are used to help shape a predictable understanding of immunity. By utilizing the increasing power of genomic, proteomic imaging, and other technologies, it is possible to examine the immune system in a comprehensive manner in the steady state and after infectious or vaccine perturbations. These data can help generate more complete parts lists that sit at the base of modeling efforts. With these lists in hand, it is reasonable to employ omic scale data acquisition during immune responses to infer connectivity, using variation in such responses to build these putative networks (Tsang 2015). The new tools of facile genomic manipulation, CRISPR in particular, will permit rapid in vivo testing of predicted novel components, especially network hubs that may play central roles in orchestrating responses. In such studies, attention needs to be paid to quantification, doing dose-response studies and not single-point perturbation, measuring output at densely spaced points in time, and doing so by highly multiplex measurements and not examination of one or only a few parameters. This 
R.N. Germain

workflow of component discovery, interaction mapping, and quantitative evaluation will provide the inputs needed to build strong and biologically realistic models. New computational tools that make such modeling more robust and especially more accessible to noncomputationalists (Morris et al. 2010; Angermann et al. 2012; Schaff et al. 2016) will permit the field as a community to instantiate such models, conduct simulations, and test predictions using the biological systems with which they have the greatest experience. By establishing a "commons" where such models are deposited and shared, iterative refinement can be achieved as many different investigators go through the cycles of simulation and experiment. If a shared infrastructure is used for such modeling (sbml.org/ Main_Page), then initial models of limited scale can potentially be combined into more complete models that come closer and closer to a complete representation of large-scale system behavior.

If this goal is to be reached and if such an outcome can convincingly be posited to be likely to provide extremely useful guides to future vaccine design that limit trial and error and also that can help explain failures, then there needs to be a consensus achieved in the funding of efforts that provide the data sets needed for model building, which support the modeling and simulation efforts and allow the iterative testing of predictions in a virtuous cycle of experimentation and model refinement. Systems biology can only deliver on its promise to aid in vaccine design if, as a community, we believe these goals are worthy of support and we value the group efforts needed to undertake such research over a sustained period of time, while providing proper recognition for all who contribute to this critical advance in our ability to predictably influence immune behavior.

\section{ACKNOWLEDGMENTS}

I am grateful to many colleagues in the Laboratory of Immunology, the Program in Systems Immunology and Infectious Disease Modeling (PSIIM), and its successor, the Laboratory of Systems Biology, as well as those in the National
Institutes of Health (NIH) Center for Human Immunology for all their interactions and discussions over the years that contributed to many of the ideas contained in this perspective. The opinions expressed, however, are solely mine. I apologize to those investigators who important publications on relevant topics I was unable to cite because of space limitations. This work is supported by the Intramural Research program of the National Institute of Allergy and Infectious Diseases (NIAID), NIH.

\section{REFERENCES}

Altan-Bonnet G, Germain RN. 2005. Modeling T cell antigen discrimination based on feedback control of digital ERK responses. PLoS Biol 3: e356.

Altschuler SJ, Wu LF. 2010. Cellular heterogeneity: Do differences make a difference? Cell 141: 559-563.

Angermann BR, Klauschen F, Garcia AD, Prustel T, Zhang F, Germain RN, Meier-Schellersheim M. 2012. Computational modeling of cellular signaling processes embedded into dynamic spatial contexts. Nat Methods 9: 283-289.

Butcher EC, Berg EL, Kunkel EJ. 2004. Systems biology in drug discovery. Nat Biotechnol 22: 1253-1259.

Das J, Ho M, Zikherman J, Govern C, Yang M, Weiss A, Chakraborty AK, Roose JP. 2009. Digital signaling and hysteresis characterize Ras activation in lymphoid cells. Cell 136: 337-351.

Dintzis HM, Dintzis RZ, Vogelstein B. 1976. Molecular determinants of immunogenicity: The immunon model of immune response. Proc Natl Acad Sci 73: 3671-3675.

Feinerman O, Veiga J, Dorfman JR, Germain RN, AltanBonnet G. 2008. Variability and robustness in T cell activation from regulated heterogeneity in protein levels. Science 321: 1081-1084.

Gaucher D, Therrien R, Kettaf N, Angermann BR, Boucher G, Filali-Mouhim A, Moser JM, Mehta RS, Drake DR III, Castro E, et al. 2008. Yellow fever vaccine induces integrated multilineage and polyfunctional immune responses. J Exp Med 205: 3119-3131.

Germain RN. 2001. The art of the probable: System control in the adaptive immune system. Science 293: 240-245.

Germain RN. 2010. Vaccines and the future of human immunology. Immunity 33: 441-450.

Germain RN, Meier-Schellersheim M, Nita-Lazar A, Fraser ID. 2011. Systems biology in immunology: A computational modeling perspective. Annu Rev Immunol 29: 527585.

Gottschalk RA, Martins AJ, Angermann BR, Dutta B, Ng CE, Uderhardt S, Tsang JS, Fraser ID, Meier-Schellersheim M, Germain RN. 2016. Distinct NF- $\kappa B$ and MAPK activation thresholds uncouple steady-state microbe sensing from anti-pathogen inflammatory responses. Cell Syst 2: 378-390.

Hagan T, Nakaya HI, Subramaniam S, Pulendran B. 2015. Systems vaccinology: Enabling rational vaccine design 
Will Systems Biology Develop New or Improved Vaccines?

with systems biological approaches. Vaccine 33: 52945301.

Kleinstein SH, Jaswinder PS. 2001. Toward quantitative simulation of germinal center dynamics: Biological and modeling insights from experimental validation. J Theoret Biol 211: 253-275.

Li S, Nakaya HI, Kazmin DA, Oh JZ, Pulendran B. 2013. Systems biological approaches to measure and understand vaccine immunity in humans. Semin Immunol 25: 209-218.

Meyer-Hermann ME. 2006. An analysis of B cell selection mechanisms in germinal centers. Math Med Biol 23: 255277.

Minor PD. 2015. Live attenuated vaccines: Historical successes and current challenges. Virology 479-480: 379-392.

Morris MK, Saez-Rodriguez J, Sorger PK, Lauffenburger DA. 2010. Logic-based models for the analysis of cell signaling networks. Biochemistry 49: 3216-3224.

Nakaya HI, Wrammert J, Lee EK, Racioppi L, Marie-Kunze S, Haining WN, Means AR, Kasturi SP, Khan N, Li GM, et al. 2011. Systems biology of vaccination for seasonal influenza in humans. Nat Immunol 12: 786-795.

Oberg AL, Kennedy RB, Li P, Ovsyannikova IG, Poland GA. 2011. Systems biology approaches to new vaccine development. Curr Opin Immunol 23: 436-443.
Perrin H, Canderan G, Sékaly RP, Trautmann L. 2010. New approaches to design HIV-1 T-cell vaccines. Curr Opin HIV AIDS 5: 368-376.

Pulendran B, Li S, Nakaya HI. 2010. Systems vaccinology. Immunity 33: 516-529.

Querec T, Bennouna S, Alkan S, Laouar Y, Gorden K, Flavell R, Akira S, Ahmed R, Pulendran B. 2006. Yellow fever vaccine YF-17D activates multiple dendritic cell subsets via TLR2, 7, 8, and 9 to stimulate polyvalent immunity. $J$ Exp Med 203: 413-424.

Schaff JC, Vasilescu D, Moraru II, Loew LM, Blinov ML. 2016. Rule-based modeling with virtual cell. Bioinformatics 32: 2880-2882.

Schiller JT, Lowy DR. 2015. Raising expectations for subunit vaccine. J Infect Dis 211: 1373-1375.

Tas JM, Mesin L, Pasqual G, Targ S, Jacobsen JT, Mano YM, Chen CS, Weill JC, Reynaud CA, Browne EP, et al. 2016. Visualizing antibody affinity maturation in germinal centers. Science 351: 1048-1054.

Trautmann L, Sekaly RP. 2011. Solving vaccine mysteries: A systems biology perspective. Nat Immunol 12: 729-731.

Tsang JS. 2015. Utilizing population variation, vaccination, and systems biology to study human immunology. Trends Immunol 36: 479-493. 


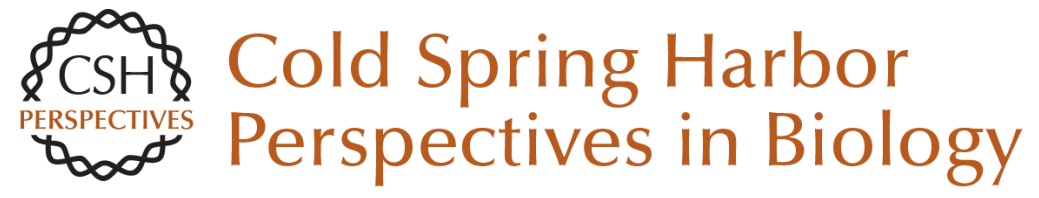

\title{
Will Systems Biology Deliver Its Promise and Contribute to the Development of New or Improved Vaccines?: What Really Constitutes the Study of "Systems Biology" and How Might Such an Approach Facilitate Vaccine Design
}

\author{
Ronald N. Germain
}

Cold Spring Harb Perspect Biol 2018; doi: 10.1101/cshperspect.a033308 originally published online October 16, 2017

\section{Subject Collection Immune Memory and Vaccines: Great Debates}

Is There Natural Killer Cell Memory and Can It Be Harnessed by Vaccination?: Can Natural Killer and CD8 T Cells Switch Jobs?

Christine A. Biron and Marcus Altfeld

Is There Natural Killer Cell Memory and Can It Be Harnessed by Vaccination?: Vaccination

Strategies Based on NK Cell and ILC Memory

Megan A. Cooper, Todd A. Fehniger and Marco Colonna

Is It Possible to Develop Cancer Vaccines to Neoantigens, What Are the Major Challenges, and How Can These Be Overcome?: Neoantigens as Vaccine Targets for Cancer

Haydn T. Kissick

Is It Possible to Develop Cancer Vaccines to Neoantigens, What Are the Major Challenges, and How Can These Be Overcome?: Neoantigens: Nothing New in Spite of the Name

Olivera J. Finn and Hans-Georg Rammensee
Is There Natural Killer Cell Memory and Can It Be Harnessed by Vaccination?: NK Cell Memory and Immunization Strategies against Infectious

Diseases and Cancer Joseph C. Sun and Lewis L. Lanier

Is There Natural Killer Cell Memory and Can It Be Harnessed by Vaccination?: Natural Killer Cells in Vaccination

Harold R. Neely, Irina B. Mazo, Carmen Gerlach, et al.

Is It Possible to Develop Cancer Vaccines to Neoantigens, What Are the Major Challenges, and How Can These Be Overcome?: Targeting the Right Antigens in the Right Patients Stephen P. Schoenberger

Which Dengue Vaccine Approach Is the Most Promising, and Should We Be Concerned about Enhanced Disease after Vaccination?: There Is Only One True Winner Scott B. Halstead

For additional articles in this collection, see http://cshperspectives.cshlp.org/cgi/collection/

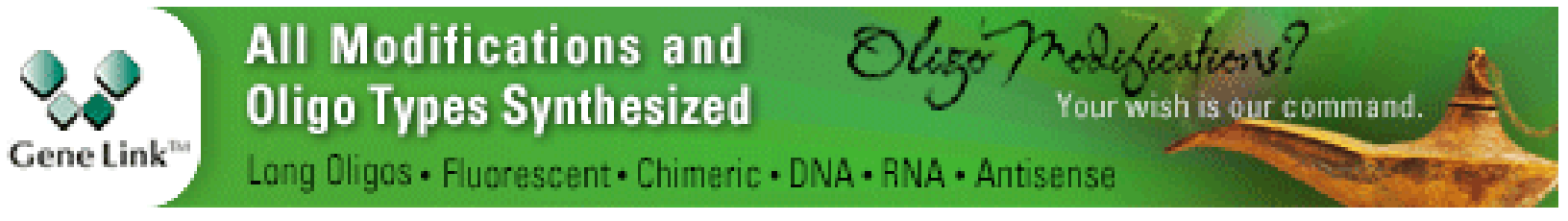


Which Dengue Vaccine Approach Is the Most Promising, and Should We Be Concerned about Enhanced Disease after Vaccination?: The Challenges of a Dengue Vaccine

Gavin Screaton and Juthathip Mongkolsapaya

Which Dengue Vaccine Approach Is the Most Promising, and Should We Be Concerned about Enhanced Disease after Vaccination?: The Path to a Dengue Vaccine: Learning from Human Natural Dengue Infection Studies and Vaccine Trials Aravinda M. de Silva and Eva Harris

Is It Possible to Develop a "Universal" Influenza Virus Vaccine?: Potential for a Universal Influenza Vaccine James E. Crowe, Jr.

Is It Possible to Develop a "Universal" Influenza Virus Vaccine?: Outflanking Antibody Immunodominance on the Road to Universal Influenza Vaccination

Davide Angeletti and Jonathan W. Yewdell
Which Dengue Vaccine Approach Is the Most Promising, and Should We Be Concerned about Enhanced Disease after Vaccination?: Questions Raised by the Development and Implementation of Dengue Vaccines: Example of the Sanofi Pasteur Tetravalent Dengue Vaccine Bruno Guy

Which Dengue Vaccine Approach Is the Most Promising, and Should We Be Concerned about Enhanced Disease after Vaccination?: The Risks of Incomplete Immunity to Dengue Virus Revealed by Vaccination

Stephen S. Whitehead and Kanta Subbarao

Is It Possible to Develop a "Universal" Influenza Virus Vaccine?: Immunogenetic Considerations Underlying B-Cell Biology in the Development of a Pan-Subtype Influenza A Vaccine Targeting the Hemagglutinin Stem Sarah F. Andrews, Barney S. Graham, John R. Mascola, et al.

Is It Possible to Develop a "Universal" Influenza Virus Vaccine?: Potential Target Antigens and Critical Aspects for a Universal Influenza Vaccine Florian Krammer, Adolfo García-Sastre and Peter Palese

For additional articles in this collection, see http://cshperspectives.cshlp.org/cgi/collection/

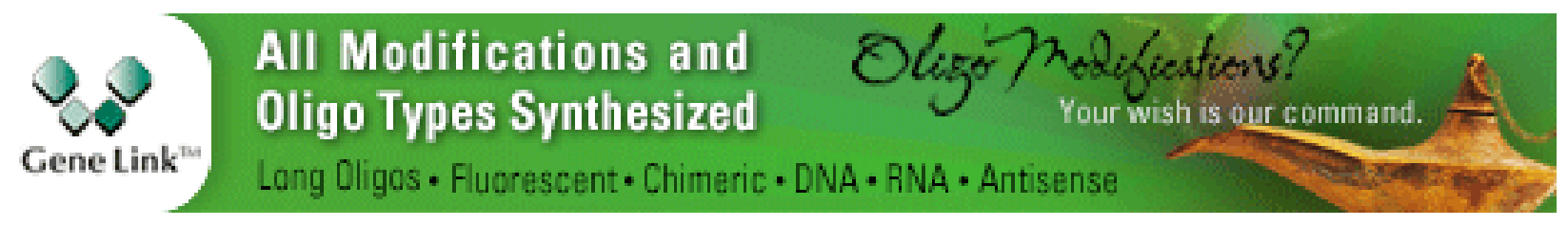

